\title{
Efficacy and Safety of Sublingual Fentanyl Tablets in Breakthrough Cancer Pain Management According to Cancer Stage and Background Opioid Medication
}

\author{
Jordi Guitart $^{1}$ - María Isabel Vargas ${ }^{2} \cdot$ Vicente De Sanctis $^{3} \cdot$ Jordi Folch $^{1}$. \\ Rafael Salazar $^{4} \cdot$ José Fuentes $^{5} \cdot$ Joan Coma $^{6} \cdot$ Julia Ferreras $^{7} \cdot$ Jordi Moya $^{8}$. \\ Albert Tomás ${ }^{9} \cdot$ Pere Estivill $^{2} \cdot$ Francisco Rodelas $^{4} \cdot$ Antonio Javier Jiménez ${ }^{10}$. \\ Almudena Sanz ${ }^{10}$
}

Published online: 23 March 2018

(C) The Author(s) 2018

\begin{abstract}
Objective Our objective was to assess the effect of sublingual fentanyl tablets (SFTs) on pain relief, quality of life, and adverse effects in patients with cancer pain, according to cancer stage and background opioid regimen. Methods Subgroup analyses from a recently completed study were performed according to cancer stage (locally advanced cancer [LAC] vs. metastatic cancer) and most frequent background opioid medication (fentanyl vs. oxycodone/naloxone). The efficacy and safety of SFTs were evaluated, recording pain intensity (PI), onset of pain relief, and adverse events (AEs). Health status was assessed with
\end{abstract}

Jordi Guitart

jordi.guitart@hospitalplato.com

1 Department of Anesthesiology, Hospital Plató, C/Plató 21, 08006 Barcelona, Spain

2 Department of Anesthesiology, Parc Sanitari Sant Joan de Deu, Barcelona, Spain

3 Pain Unit, Department of Anesthesiology, Hospital Universitari Sagrat Cor, Barcelona, Spain

4 Department of Anesthesiology, Hospital Comarcal d'Inca, Palma de Mallorca, Spain

5 Department of Anesthesiology, Pius Hospital de Valls, Tarragona, Spain

6 Department of Anesthesiology, Hospital General de l'Hospitalet, Barcelona, Spain

7 Pain Unit, Department of Anesthesiology, Hospital Residència Sant Camil, Barcelona, Spain

8 Pain Unit, Department of Anesthesiology, Hospital Mateu Orfila, Menorca, Spain

9 Pain Unit, Department of Anesthesiology, Fundació Hospital Sant Bernabé, Barcelona, Spain

10 Kyowa Kirin Farmacéutica SLU, Madrid, Spain the Short Form 12, version 2 (SF-12v2) questionnaire and the Hospital Anxiety and Depression Scale (anxiety subscale [HADS-A] and depression subscale [HADS-D]).

Results In total, $54(67.5 \%)$ patients had LAC and 26 $(32.5 \%)$ had metastatic cancer. The oxycodone/naloxone group included 39 patients $(48.1 \%)$ and the fentanyl group $29(35.8 \%)$. In all subgroups, pain relief was achieved within $5 \mathrm{~min}$ in an increasing number of individuals over time; at the end of the study, PI values decreased (PI-end: $44.4 \%$ for LAC vs. $57.9 \%$ for metastatic cancer; $44.4 \%$ for fentanyl vs. $38.6 \%$ for oxycodone/naloxone). HADS and mental component summary (MCS) SF-12v2 scores significantly improved in the LAC group (HADS-A 9.44-8.04; HADS-D 10.46-8.15; MCS 44.69-45.94) and in the fentanyl group (HADS-A 10.05-8.33; HADS-D 11.95-8.76; MCS 44.38-47.19). AEs were reported in few patients and were mostly mild.

Conclusions Exploratory subgroup analyses show the efficacy and safety of SFTs for the treatment of breakthrough pain in patients with cancer, regardless of their cancer stage and background opioid medication.

\section{Key Points}

Sublingual fentanyl tablets could represent an effective and safe treatment option to control the transient exacerbation of pain in patients with advanced cancer, either locally advanced or metastatic.

This work suggests the relevance of the optimization of background pain treatment to alleviate breakthrough pain episodes. 


\section{Introduction}

Pain is one of the most common and frequent distressing symptoms in patients with cancer and intensifies as the disease progresses. In addition to persistent pain, patients may also experience transient exacerbations of significant and severe pain on a background of otherwise well-controlled pain [1-3]. These sudden, temporary flares of severe pain are called breakthrough pain (BTP) as the pain "breaks through" the long-acting medication prescribed to treat cancer pain. The duration of the pain is short, typically lasting for $30-45 \mathrm{~min}$, with several episodes per day [4].

BTP is associated with increased emotional distress and a risk of developing anxiety and depression. Because of the negative consequences of BTP on a patient's quality of life (QoL) and mental health, effective BTP management is required. The choice of adequate and fast-acting pharmacological strategies becomes crucial in controlling cancerrelated BTP.

Optimal pain management interventions depend on a variety of patient-related and pain-related factors, including disease stage and patient performance status [3]. Depending on the type and extent of the cancer, the administration routes may be limited for some patients, and innovative methods may need to be utilized [5]. Optimizing the background analgesic regimen can also play an important role in the management of cancer BTP and should be considered at all stages of the patient's illness [6].

Sublingual fentanyl tablets (SFTs) are a non-invasive mechanism for immediate drug absorption through the sublingual mucosa and provide rapid onset of pain relief compared with traditional oral dosing [7-9]. Moreover, treatment of BTP with SFTs in patients with cancer has proven well-tolerated and to potentially enhance QoL [10-12]. However, to our knowledge, no studies have investigated the effect of SFTs in BTP management according to relevant factors such as disease stage and background treatment regimen.

To provide such information, we conducted subgroup analyses on data from a recently completed trial contributing to the assessment of the effect of SFTs on QoL and pain relief in patients with cancer pain [13]. Subgroup analyses were performed to determine whether the cancer stage (locally advanced cancer [LAC] vs. metastatic cancer) affected response to SFTs. We also performed subgroup analyses to compare the effect of SFTs in patients treated with one of the two pharmacological opioids most frequently used to control chronic pain: transdermal fentanyl and oral oxycodone/naloxone. We hypothesized that an appropriate analgesic effect of SFTs for BTP control might be subject to the background treatment option.

The analyses were carried out to explore possible differences in how these subsets of patients responded to SFTs in terms of QoL, pain relief outcomes, and treatment-related adverse effects.

\section{Methods}

\subsection{Study Design}

A multicenter, prospective, observation post-authorization open-label study was previously conducted at nine pain units in Catalonia and the Balearic Islands in Spain between March and December 2013. The methods of this trial are described in detail elsewhere [13]. In brief, patients who met eligibility criteria (Sect. 2.2) provided data on their prestudy (baseline) health, treatment, pain, and QoL at the screening visit. All outcomes were assessed at 3 (visit 1), 7 (visit 2), 15 (visit 3), and 30 days (end of study) after starting the treatment. For each BTP episode, patients self-administered SFTs (Abstral, Kyowa Kirin Farmacéutica SLU, Madrid, Spain). The initial SFT dose was determined by the clinician based on prior treatment for BTP and with consideration of the opioid dose for background pain. The dose was then titrated up to successful analgesia $(100,200,300,400,600,800 \mu \mathrm{g})$. Changes in SFT dose were recorded throughout the study.

\subsection{Study Population}

Eligible patients were male and female adults with a confirmed diagnosis of cancer who were regularly experiencing episodes of BTP that were partially relieved (scored $\geq 6$ on an 11-point numerical rating scale [NRS]). Patients were required to be opioid tolerant and receiving a fixed-dose schedule of opioids equivalent to oral morphine $\geq 60 \mathrm{mg} / \mathrm{day}$, or transdermal fentanyl $25 \mu \mathrm{g} / \mathrm{h}$, oral oxycodone $30 \mathrm{mg} / \mathrm{day}$, oral hydromorphone $8 \mathrm{mg} /$ day, oral oxymorphone $25 \mathrm{mg} /$ day, or an equianalgesic dose of any other opioid.

Patients were excluded if they had hypersensitivities or allergies to fentanyl or to any of the other excipients of the study drug, had a history of alcohol or substance abuse, or had neurologic or psychiatric impairment that could compromise study data collection.

\subsection{Efficacy and Safety Assessments}

The effect of BTP medication with SFTs in patients with cancer pain was evaluated primarily on two parameters: pain relief and QoL. Pain was assessed using a generic 
unidimensional pain questionnaire, the 11-item NRS, in which patients rated their pain intensity (PI) from 0 (no pain) to 10 (worst pain imaginable). Pain relief was assessed by evaluating the time to onset of analgesia and the time to maximum analgesic effect following the administration of SFTs. At each clinical visit, each patient also reported the number of irruptive pain episodes and the duration of each episode. The health status instrument used to evaluate patient health-related QoL was the Short Form 12 (version 2) questionnaire (SF-12v2), which uses 12 questions to measure functional health and well-being from the patient's perspective. It comprises the physical component summary (PCS) and the mental component summary (MCS) [14, 15], which range from 0 (lowest level of health) to 100 (highest level of health). The Hospital Anxiety and Depression Scale (HADS) questionnaire, consisting of seven statements on the anxiety subscale (HADS-A) and seven on the depression subscale (HADSD) $[16,17]$, was also applied to detect clinically significant cases of anxiety or depression. Safety and tolerability were assessed based on patients' and clinicians' reports of adverse events (AEs).

\subsection{Statistical Analysis}

We identified subgroups of patients in which the effect of SFTs on the efficacy and safety outcomes might be relevant. Subgroup analyses were then performed according to cancer stage (LAC vs. metastatic cancer) and most frequent pharmacological treatment option for background pain (fentanyl vs. oxycodone/naloxone). For each subgroup, demographics and disease-related features were analyzed descriptively using frequencies, means \pm standard deviations (SDs), as appropriate. The SF-12 PCS and MCS scores were computed as normalized scores (mean $50 \pm 10$ ). Statistical analyses were conducted using the Chi squared test for categorical data and the paired twotailed $t$ test or analysis of variance (ANOVA) for continuous data; $p$ values $<0.05$ were considered statistically significant, with no adjustments for multiplicity. All statistical analyses were performed using SAS statistical software version 9.2 (SAS Institute Inc., Cary, NC, USA).

\section{Results}

Flow of patients through the study, as well as patient demographics and other baseline characteristics, are summarized elsewhere [13]. Briefly, 102 patients were considered eligible, 81 were enrolled, and 69 completed the 30-day observation period. The mean age was 69.7 years (range 40-91), and $58.0 \%$ of patients were female. LACs were found in 54 patients $(67.5 \%)$ and metastatic cancers in $26(32.5 \%)$. The most frequent opioid pain medications taken by patients for background pain were oxycodone/naloxone and fentanyl (in 39 patients [48.1\%] and 29 [35.8\%], respectively). Patient demographics for the analyzed subgroups are summarized in Table 1.

\subsection{Efficacy Endpoints}

Main pain intensity outcomes throughout the study for each subgroup of patients are shown in Table 2. Levels of PI improved significantly compared with baseline for all assessment points and all subgroups $(p<0.05)$. At the end of the study, the mean PI-start improved significantly $(p<0.0001)$ compared with baseline in both the metastatic cancer group and the LAC group (29.2 and $27.6 \%$ of improvement, respectively). The mean PI-end reduced 2.19 points in patients with LAC (44.4\% of improvement) and 2.27 in patients with metastatic cancer $(57.9 \%$ of improvement). According to the background treatment regimen, the mean PI-start also improved significantly $(p<0.0001)$ compared with baseline in both fentanyl and oxycodone/naloxone groups (32.1 and 24.3\%, respectively). The mean PI-end improved $44.4 \%$ in patients taking fentanyl versus $38.6 \%$ in patients receiving oxycodone/naloxone.

Most patients experienced one to five daily episodes at the end of the study for all analyzed subgroups: $52(94.5 \%)$ and $22(88.0 \%)$ patients in the LAC and metastatic groups, respectively, and $23(95.8 \%)$ and $19(82.6 \%)$ in the fentanyl and oxycodone/naloxone groups, respectively (Fig. 1).

Figure 2 shows the percentage of patients according to the average duration of BTP episodes at baseline and at the end of the study, for all subgroups. In all of them, the proportion of patients with BTP episodes of more than 15 min was reduced at the end of the study (visit 4) as compared with baseline. At the end of the study, a higher proportion of patients in the LAC group experienced shorter pain duration of BTP episodes than patients in the metastatic group (75.0 vs. $66.7 \%$ reported episodes lasting $<10 \mathrm{~min}$ ) (Fig. 2a). According to the background pain treatment, the percentage of patients who experienced shorter BTP episodes was higher in the fentanyl group than in the oxycodone/naloxone group (76.2 vs. 54.5\% reported episodes lasting $<10 \mathrm{~min}$ ) (Fig. 2b).

In all subgroups, an increasing number of patients over time experienced pain relief within an average of $5 \mathrm{~min}$. At the end of the study assessment, the mean time to onset of relief following SFT administration was $<5 \mathrm{~min}$ in $41.7 \%$ of patients with LAC and $47.6 \%$ of patients with metastatic cancer (Fig. 3a) and in 42.9 and $40.9 \%$ of patients receiving fentanyl and oxycodone/naloxone, respectively (Fig. 3b). At this visit, the time to first effect following SFT 
Table 1 Patient demographics for the analyzed subgroups

\begin{tabular}{lllll}
\hline Characteristic & LAC & Metastatic cancer & Fentanyl & Oxycodone/naloxone \\
\hline Age (years) & $22(70.4 \pm 13.2)$ & $54(70.2 \pm 11.9)$ & $27(67.7 \pm 12.3)$ & $24(72.5 \pm 10.5)$ \\
Sex & & & & \\
$\quad$ Male & $8(34.8)$ & $21(36.2)$ & $15(51.7)$ & $15(62.5)$ \\
Female & $15(65.2)$ & $37(63.8)$ & $14(48.3)$ & $9(37.5)$ \\
Weight $(\mathrm{kg})$ & $20(65.1 \pm 13.7)$ & $53(72.1 \pm 13.9)$ & $26(68.0 \pm 12.4)$ & $21(73.7 \pm 14.7)$ \\
\hline
\end{tabular}

Data are presented as $n(\%)$ or $N$ (mean \pm standard deviation)

LAC locally advanced cancer

\begin{tabular}{|c|c|c|c|c|}
\hline \multirow[t]{2}{*}{ Subgroup } & \multicolumn{2}{|l|}{ PI-start } & \multicolumn{2}{|l|}{ PI-end } \\
\hline & Mean \pm SD & Reduction (\%) & Mean $\pm \mathrm{SD}$ & Reduction (\%) \\
\hline \multicolumn{5}{|l|}{ LAC } \\
\hline Baseline & $8.69 \pm 1.00$ & & $4.93(2.15)$ & \\
\hline Visit 4 & $6.29 \pm 1.84^{*}$ & 27.6 & $2.74(1.82)^{*}$ & 44.4 \\
\hline \multicolumn{5}{|c|}{ Metastatic cancer } \\
\hline Baseline & $8.28 \pm 0.94$ & & $3.92(1.35)$ & \\
\hline Visit 4 & $5.86 \pm 1.53^{*}$ & 29.2 & $1.65(1.27)^{*}$ & 57.9 \\
\hline \multicolumn{5}{|l|}{ Fentanyl } \\
\hline Baseline & $8.63 \pm 1.21$ & & $4.96(2.22)$ & \\
\hline Visit 4 & $5.86 \pm 1.74^{*}$ & 32.1 & $2.76(1.70)^{*}$ & 44.4 \\
\hline \multicolumn{5}{|c|}{ Oxycodone/naloxone } \\
\hline Baseline & $8.65 \pm 0.88$ & & 4.35 (1.75) & \\
\hline Visit 4 & $6.55 \pm 1.53^{*}$ & 24.3 & $2.67(1.56)^{*}$ & 38.6 \\
\hline
\end{tabular}

LAC locally advanced cancer, PI-start pain intensity at the start of the breakthrough pain episode, PI-end pain intensity at the end of the breakthrough pain episode, $S D$ standard deviation

$* p<0.0001$
Table 2 Pain intensity outcomes based on an 11-point numerical rating scale for each subgroup of patients administration was within $10 \mathrm{~min}$ in 66.7 and $95.2 \%$ of patients according to cancer stage (LAC and metastatic, respectively) and in 66.7 and $72.7 \%$ of patients according to background pain treatment (fentanyl and oxycodone/naloxone, respectively).

No relevant differences were found in the number of irruptive pain episodes in the analyzed subgroups (data not shown).

The results obtained with the SF-12v2 questionnaire at baseline and at the end of the study, as well as the scores registered on the HADS-A and HADS-D subscales, according to cancer stage and pharmacological treatment option for background pain, are presented in Tables 3 and 4 , respectively. No significant improvements were observed in the SF-12v2 PCS scores in the subgroups of patients defined by cancer stage. After 30 days, SF-12v2 MCS scores showed a slight increase compared with baseline in both groups, although these results were only significant in the LAC group (baseline-MCS 44.69, end-ofstudy-MCS 45.94; $p=0.0243$ ). The mean \pm SD HADS-A subscale score was $9.44 \pm 3.23$ at baseline and
$8.04 \pm 2.77$ at the end of the study for the LAC group $(p<0.0001) ; 8.29 \pm 3.12$ at baseline and $7.24 \pm 3.51$ at the end of the study for the metastatic cancer group ( $p=0.1014)$. HADS-D scores only decreased significantly in the LAC group (baseline 10.46, end-of-study 8.15; $p=0.0009$ ).

No significant improvements were observed in the SF$12 \mathrm{v} 2$ PCS scores in the subgroups of patients defined by pharmacological treatment option for background pain. At the end of the study, SF-12v2 MCS scores increased compared with baseline in both groups, although results were significant only in the fentanyl group (baseline-MCS 44.38, end-of-study-MCS 47.19; $p=0.0332$ ). Patients receiving fentanyl showed significant improvement in the HADS-A score (baseline 10.05, end-of-study 8.33; $p=0.0016)$, as did those receiving oxycodone/naloxone treatment (baseline 9.16, end-of-study 7.61; $p=0.0226$ ). HADS-D scores significantly decreased in the fentanyl group (baseline 11.95, end-of-study 8.76; $p=0.0013$ ). 


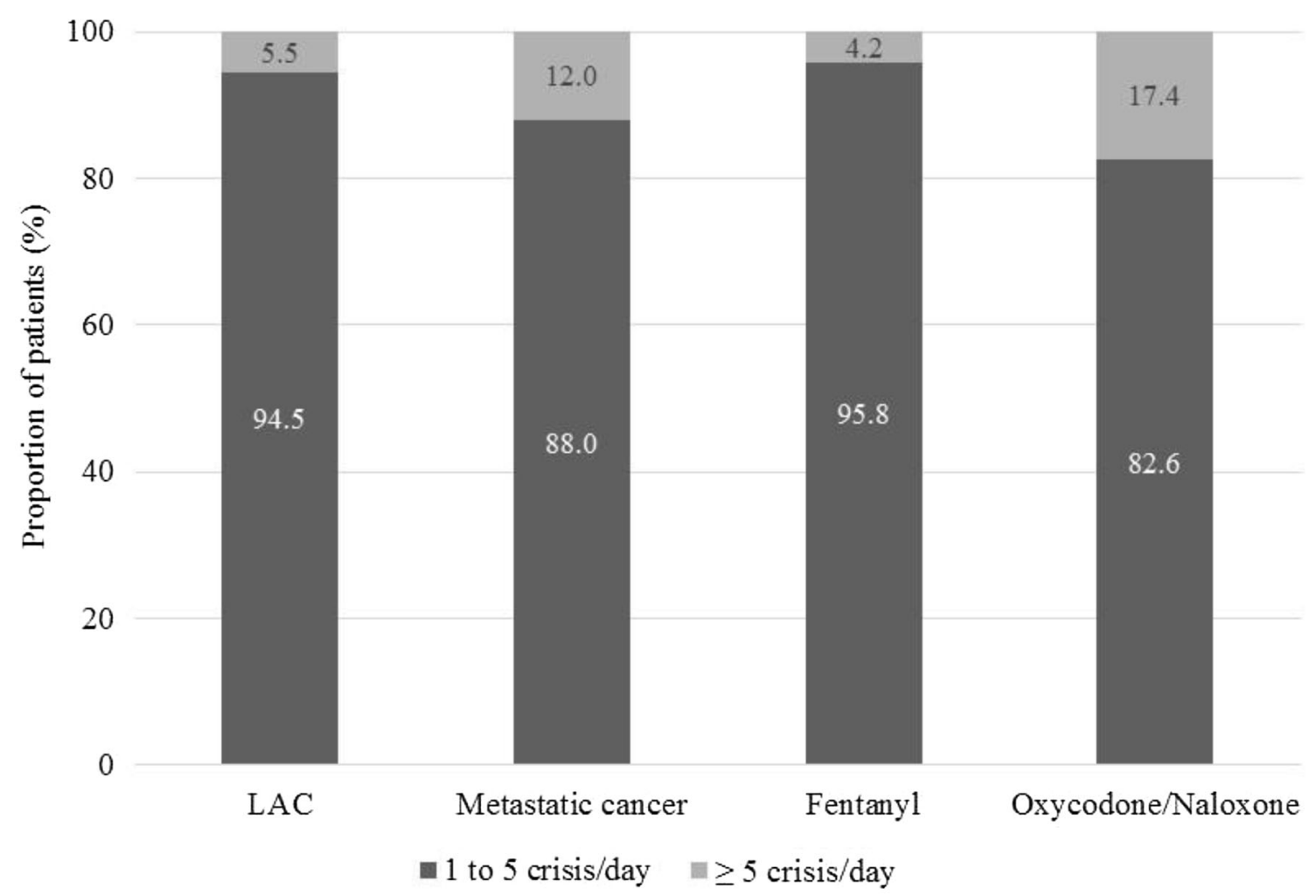

Fig. 1 Proportion of patients experiencing daily breakthrough pain episodes at the end of the study for all subgroups. LAC locally advanced cancer

\subsection{Safety Endpoints}

Table 5 shows the percentage of patients presenting AEs related to SFT treatment for each subgroup. AEs were reported at the end of the study in a minority of patients $(18.8,38.1,28.6$, and $13.6 \%$ in LAC, metastatic cancer, fentanyl, and oxycodone/naloxone groups, respectively). Table 6 describes the type and severity of AEs at the end of the study visit for each subgroup of patients. Most AEs were mild ( 26 of 38 total AEs), 12 of 38 were moderate, and none were severe. The most frequently reported was constipation (19 of 38), followed by somnolence (7 of 38), nausea (5 of 38), vomiting (5 of 38), and skin disorders (2 of 38). Constipation was described in $8.6 \%$ of patients in the LAC group, $30.8 \%$ in the metastatic cancer group, $16.7 \%$ in the fentanyl group, and $8.0 \%$ in the oxycodone/naloxone group.

\section{Discussion}

The present study was conducted to investigate whether sublingual fentanyl formulation is equally effective in terms of pain relief, QoL, and safety for the treatment of BTP at different stages of cancer, and under different opioid regimens for background pain. To address these questions, we analyzed data from a recently completed trial contributing to the assessment of the effect of SFTs in patients with cancer, by conducting subgroup analyses based on cancer stage and treatment of background pain.

After treatment with SFTs, pain relief was significantly achieved in all subgroups analyzed. Compared with the first visit, at 30 days there was a substantial increment of individuals experiencing pain relief within $5 \mathrm{~min}$, indicating the effective treatment of BTP episodes with SFTs over time.

According to the background treatment, improvement of PI values at the end of the study compared with baseline were higher in the fentanyl group (PI-start 32.1 vs. 24.3\%; PI-end 44.4 vs. $38.6 \%$ ). These results suggest that patients already taking fentanyl for background pain can have a better response to SFTs than those receiving oxycodone/naloxone. When patients were grouped according to cancer stage, PI outcomes showed a greater improvement in patients with metastatic cancer than in the LAC group (PI-start 29.2 vs. 27.6\%; PI-end 44.4 vs. $57.9 \%$ ), suggesting that SFTs can relieve BTP episodes in patients with a complicated cancer stage.

In addition, 95\% of patients with metastatic cancer experienced an onset of action of SFTs in $<10 \mathrm{~min}$ compared with $66.7 \%$ of patients with LAC. These results are very encouraging since-although it occurs during all 


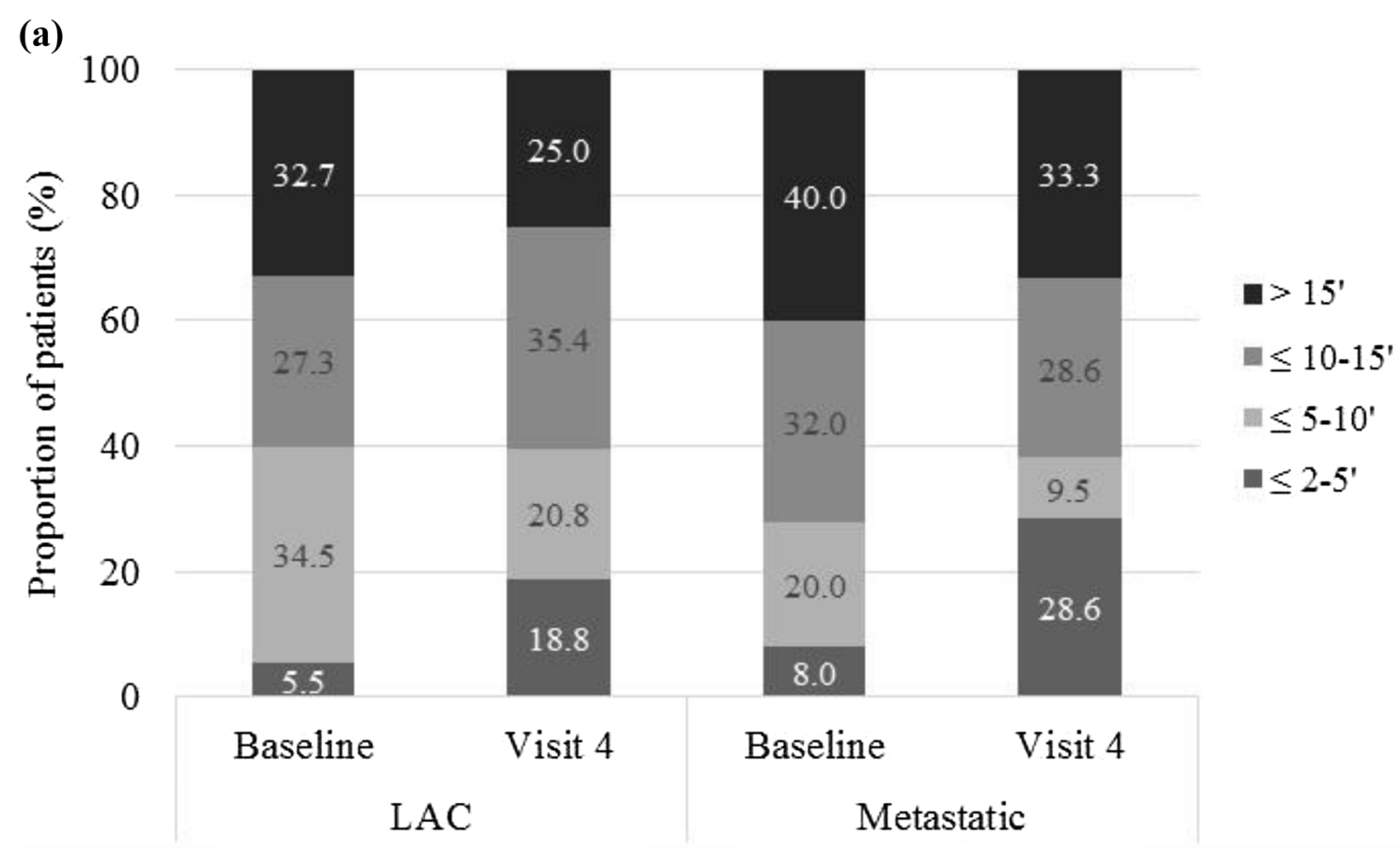

(b)

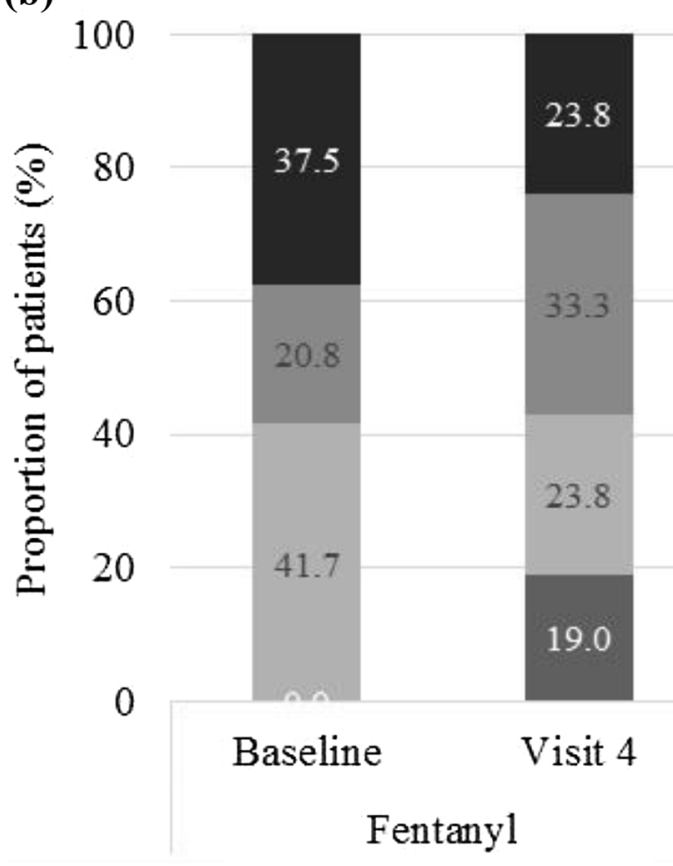

Fig. 2 Average duration of breakthrough pain episodes at baseline and at the end of the study, following administration of sublingual fentanyl tablets (SFTs) in subgroup of patients according to a cancer

stages of cancer-BTP has been described as more frequently experienced by patients with advanced disease [18]. However, in our study, BTP episodes were more frequently registered in patients with LAC (data not shown).

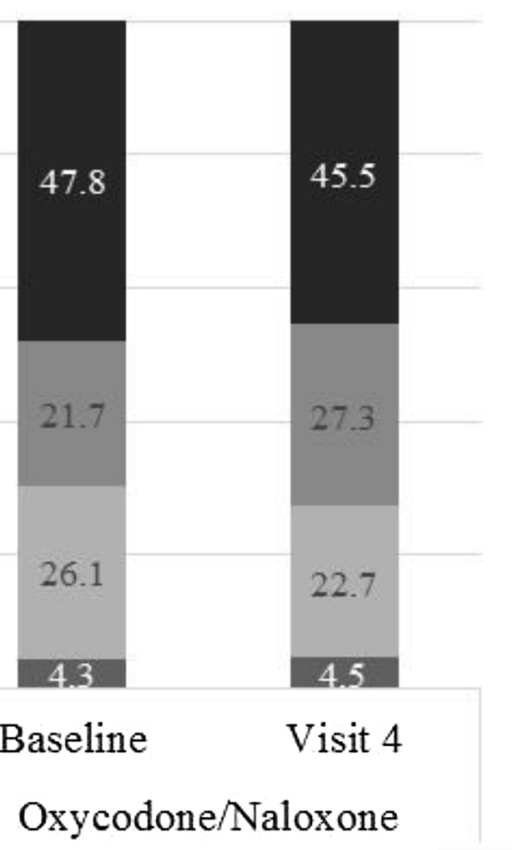

- $>15^{\prime}$

$\square \leq 10-15^{\prime}$

$=5-10^{\prime}$

घ $\leq 2-5^{\prime}$

stage and $\mathbf{b}$ pharmacological treatment option for background pain. $L A C$ locally advanced cancer

When exploring the duration of BTP episodes in each subgroup of patients, we observed that a higher percentage of patients in the metastatic group than in the LAC group (28.6 vs. $18.8 \%$ ) experienced BTP episodes that lasted $<5$ min. Still, most patients in both groups experienced BTP events that lasted $<15 \mathrm{~min}$. These episodes were 
Fig. 3 Percentage of patients versus average time to onset of pain relief at the end of the study, following administration of sublingual fentanyl tablets (SFTs) in subgroup of patients according to a cancer stage and b pharmacological treatment option for background pain. $L A C$ locally advanced cancer

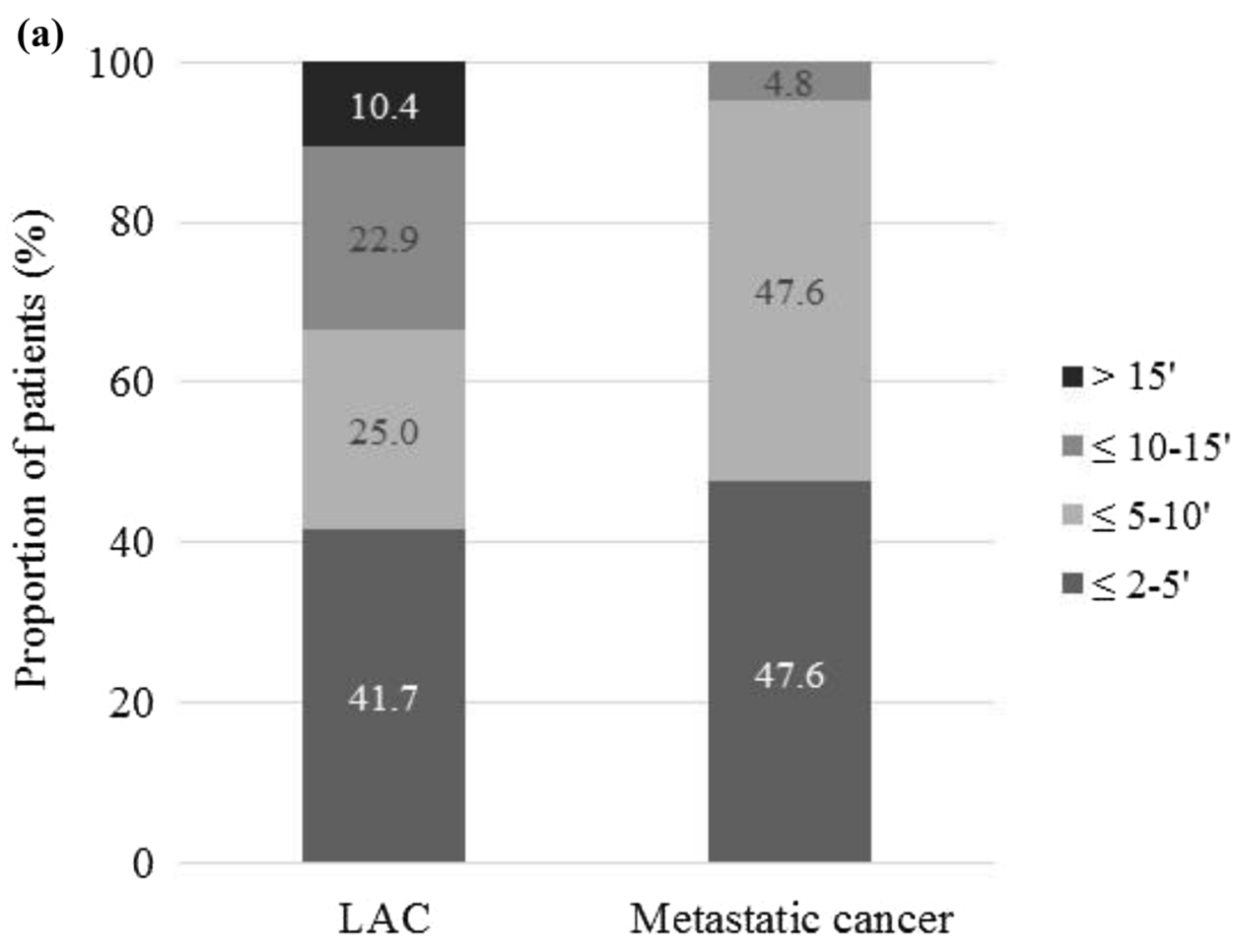

(b)

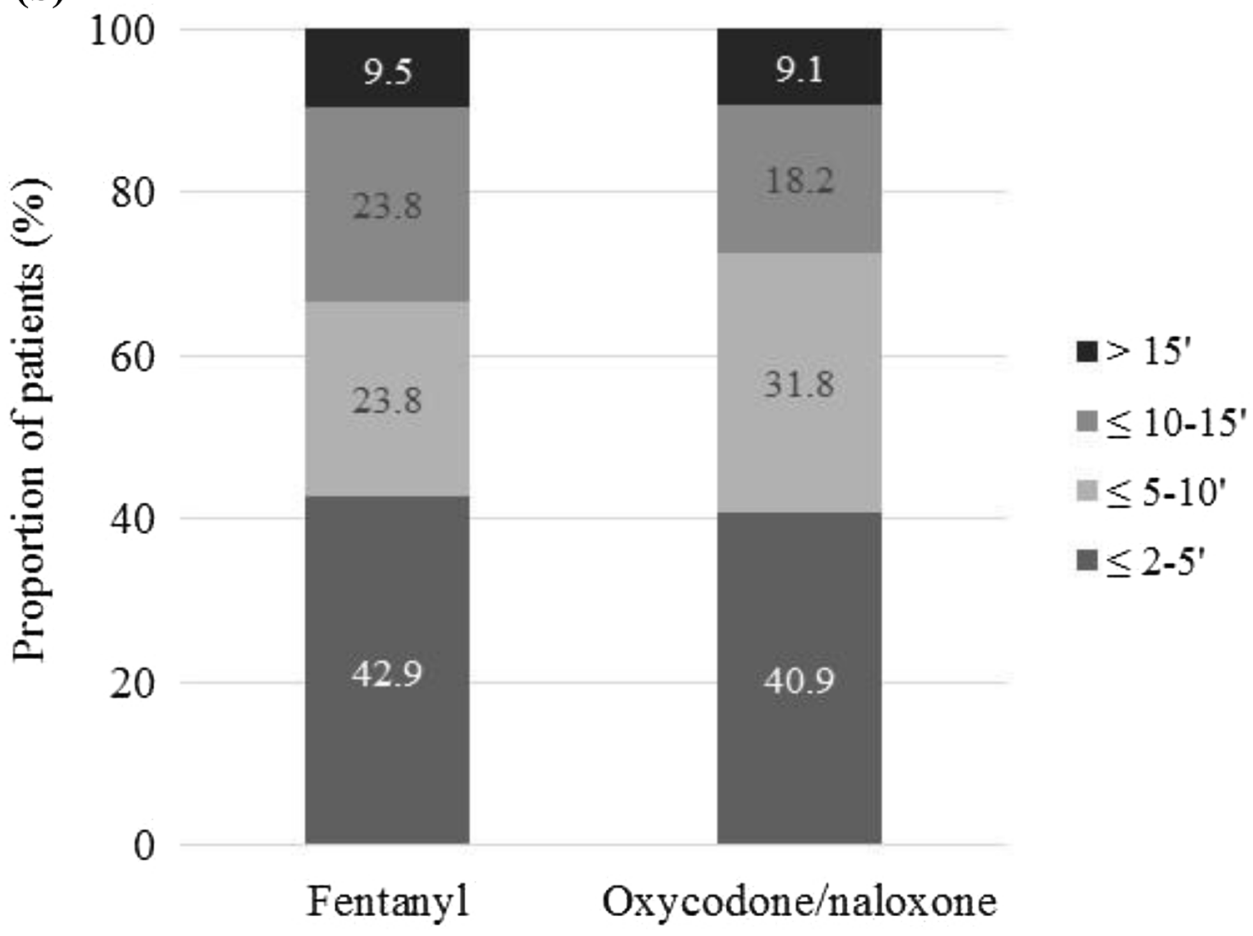

shorter in the fentanyl group than in the oxycodone/naloxone group, suggesting a relationship between the background analgesic regimen and the duration of BTP episodes. Nonetheless, as mentioned, pain relief with SFTs was equally effective in both groups.
Along with pain, depression and anxiety are associated with decreased health-related QoL in patients with cancer [19]. In this study, the results from the HADS questionnaire revealed improvements in both anxiety and depression subscales in patients of all subgroups after treatment with SFTs. Reductions in these troubling and disabling 
Table 3 Short Form-12 component summary and Hospital Anxiety and Depression Scale outcomes scores, for subgroups of patients defined by cancer stage
Table 4 Short Form-12 component summary and Hospital Anxiety and Depression Scale outcomes scores, for subgroups of patients defined by pharmacological treatment option for background pain

\begin{tabular}{|c|c|c|c|c|c|c|}
\hline \multirow[t]{2}{*}{ Scale/component } & \multicolumn{3}{|l|}{ LAC } & \multicolumn{3}{|c|}{ Metastatic cancer } \\
\hline & Baseline & End of study & $p$ value & Baseline & End of study & $p$ value \\
\hline SF-12v2 PCS & $38.14 \pm 4.71$ & $37.80 \pm 4.59$ & 0.4917 & $37.42 \pm 5.28$ & $36.87 \pm 4.88$ & 0.7762 \\
\hline SF-12v2 MCS & $44.69 \pm 6.40$ & $45.94 \pm 6.39$ & $0.0243 *$ & $44.70 \pm 7.00$ & $47.22 \pm 6.20$ & 0.2650 \\
\hline HADS-A & $9.44 \pm 3.23$ & $8.04 \pm 2.77$ & $<0.0001 *$ & $8.29 \pm 3.12$ & $7.24 \pm 3.51$ & 0.1014 \\
\hline HADS-D & $10.46 \pm 4.26$ & $8.15 \pm 4.08$ & $0.0009^{*}$ & $10.24 \pm 5.52$ & $8.95 \pm 5.21$ & 0.4435 \\
\hline
\end{tabular}

Data are presented as mean \pm standard deviation unless otherwise indicated

$H A D S-A$ Hospital Anxiety and Depression Scale-anxiety subscale, HADS-D Hospital Anxiety and Depression Scale-depression subscale, LAC locally advanced cancer, MCS mental component summary, $P C S$ physical component summary, $S F-12 v 2$ Short Form 12 (version 2) $* p<0.05$

\begin{tabular}{|c|c|c|c|c|c|c|}
\hline \multirow[t]{2}{*}{ Scale/component } & \multicolumn{3}{|l|}{ Fentanyl } & \multicolumn{3}{|c|}{ Oxycodone/naloxone } \\
\hline & Baseline & End of study & $p$ value & Baseline & End of study & $p$ value \\
\hline SF-12v2 PCS & $37.80 \pm 5.22$ & $36.79 \pm 5.26$ & 0.3719 & $37.69 \pm 4.98$ & $38.03 \pm 4.46$ & 0.8103 \\
\hline SF-12v2 MCS & $44.38 \pm 6.46$ & $47.19 \pm 6.21$ & $0.0332 *$ & $44.31 \pm 6.33$ & $45.61 \pm 6.80$ & 0.4184 \\
\hline HADS-A & $10.05 \pm 2.98$ & $8.33 \pm 2.58$ & $0.0016 *$ & $9.16 \pm 3.37$ & $7.61 \pm 2.97$ & $0.0226 *$ \\
\hline HADS-D & $11.95 \pm 4.20$ & $8.76 \pm 3.67$ & $0.0013 *$ & $9.26 \pm 3.18$ & $8.00 \pm 4.35$ & 0.5610 \\
\hline
\end{tabular}

Data are presented as mean \pm standard deviation unless otherwise indicated

$H A D S-A$ Hospital Anxiety and Depression Scale-anxiety subscale, HADS-D Hospital Anxiety and Depression Scale-depression subscale, LAC locally advanced cancer, MCS mental component summary, $P C S$ physical component summary, $S F-12 v 2$ Short Form 12 (version 2)

$* p<0.05$
Table 5 Number of patients presenting treatment-related adverse events for each subgroup of patients

\begin{tabular}{lrrr}
\hline Subgroup & Baseline & Visit 4 & $p$ value \\
\hline LAC & $18(32.7)$ & $9(18.8)$ & $<0.0001$ \\
Metastatic cancer & $14(53.8)$ & $8(38.1)$ & $<0.0001$ \\
Fentanyl & $10(41.7)$ & $6(28.6)$ & $<0.0001$ \\
Oxycodone/naloxone & $5(20.8)$ & $3(13.6)$ & $<0.0001$ \\
\hline
\end{tabular}

Data are presented as $N(\%)$

$L A C$ locally advanced cancer

$p$ values based on pairwise comparisons versus baseline

symptoms were clearly observed in the LAC and fentanyl groups, in which both the HADS-A and the HADS-D scores reduced significantly. Similarly, SF-12v2 MCS scores increased significantly in these two groups, and not in the other two, indicating that respondents reacted differently to treatment depending on the cancer stage and their medication for control of background pain.

The subgroup analyses showed that SFTs were generally well-tolerated in all groups, as none of the reported AEs were considered severe, although some differences were observed. The percentage of patients reporting AEs was lower in the LAC group than in the metastatic group (18.8 vs. $38.1 \%$, respectively) and in patients receiving oxycodone/naloxone than in those receiving fentanyl (13.6 vs. $28.6 \%$, respectively). Despite these differences, most AEs were mild, with constipation the most frequently reported AE.

In combination, these findings suggest that the use of rescue medication is only one aspect of the management of BTP and that cancer stage and modification of the background analgesic regimen should also be considered. Efforts to optimize background pain treatment might provide a satisfactory balance between analgesia and AEs, helping to alleviate episodes of BTP.

This study has some important limitations. First, the sample sizes were small and uneven for all subgroups, which makes interpreting the results and the clinical significance of these findings difficult. However, we divided our patients in two subgroups (different cancer stages and medications for treatment of background pain) to conduct an exploratory analysis and identify potential differences between groups. Further studies with larger samples and balanced groups should provide robust and more reliable findings on the possible implications of cancer stage and treatment of background pain in the management of BTP with SFT. Second, it is difficult to separate the effects of 
Table 6 Type and severity of treatment-related adverse events at the end of the study visit for each subgroup of patients

\begin{tabular}{|c|c|c|c|}
\hline Subgroup and adverse event & Mild & Moderate & Severe \\
\hline \multicolumn{4}{|l|}{ LAC $(n=58)$} \\
\hline Nausea & $1(1.72)$ & 0 & 0 \\
\hline Vomiting & $1(1.72)$ & 0 & 0 \\
\hline Constipation & $2(3.45)$ & $3(5.17)$ & 0 \\
\hline Somnolence & $2(3.45)$ & 0 & 0 \\
\hline Skin disorders & 0 & $1(1.72)$ & 0 \\
\hline \multicolumn{4}{|l|}{ Fentanyl $(n=24)$} \\
\hline Nausea & $1(4.17)$ & 0 & 0 \\
\hline Vomiting & $1(4.17)$ & 0 & 0 \\
\hline Constipation & $3(12.50)$ & $1(4.17)$ & 0 \\
\hline Somnolence & $1(4.17)$ & 0 & 0 \\
\hline Skin disorders & 0 & $1(4.17)$ & 0 \\
\hline \multicolumn{4}{|l|}{ Metastatic cancer $(n=26)$} \\
\hline Nausea & 0 & $2(7.69)$ & 0 \\
\hline Vomiting & $1(3.85)$ & $1(3.85)$ & 0 \\
\hline Constipation & $7(26.92)$ & $1(3.85)$ & 0 \\
\hline Somnolence & $3(11.54)$ & 0 & 0 \\
\hline Skin disorders & 0 & 0 & 0 \\
\hline \multicolumn{4}{|l|}{ Oxycodone/naloxone $(n=25)$} \\
\hline Nausea & 0 & $1(4.00)$ & 0 \\
\hline Vomiting & $1(4.00)$ & 0 & 0 \\
\hline Constipation & $1(4.00)$ & $1(4.00)$ & 0 \\
\hline Somnolence & $1(4.00)$ & 0 & 0 \\
\hline Skin disorders & 0 & 0 & 0 \\
\hline
\end{tabular}

Data are presented as $n(\%)$

$L A C$ locally advanced cancer

the SFT treatment from other factors. It could be that apparent differences observed between subgroups were the effect of potential confounders, which we presume are not distributed evenly among groups. Regarding patient demographics, although differences in the analyzed subgroups were not substantial, they could also affect patient outcomes. Confounding could have been reduced by restricting the study population to those with a specific value of the confounding variable(s). However, to allow a minimum sample size for analysis, variables such as dose ranges of long-acting opioids, as well as doses of SFT received, were not considered to define strata. Stratified analyses would be a good way to evaluate the effect of SFTs on subgroups and, in addition, it would be a way of examining or adjusting for confounding. Nevertheless, more participants would be required to yield conclusive results. Lastly, the findings of this study depend upon patients' self-report of pain and well-being, which can be influenced by a variety of psychosocial factors. The subjective nature of pain means measurement and interpretation are imprecise, and comparing outcomes between groups of patients can be complex.

Nonetheless, despite the large and variable incidence of BTP, no studies have described how to effectively treat it in patients at different cancer stages or considering their medication for background pain. The present study represents the first reported approach to the management of cancer BTP with SFTs in terms of pain relief, QoL, and safety according to cancer stage and opioid regimen for background pain.

\section{Conclusions}

The results of this study support the safety and efficacy of SFTs for the treatment of BTP in patients with cancer, regardless of disease stage and background analgesic regimen. Nonetheless, the variability observed in terms of pain relief and QoL suggests that cancer stage and modification of the background opioid medication should be considered for the management of cancer BTP.

Acknowledgements The authors thank Blanca Martínez-Garriga, who provided medical writing assistance on behalf of Trialance (www.trialance.com).

\section{Compliance with Ethical Standards}

Funding This work was supported by Kyowa Kirin Farmacéutica SLU. The authors received research funding from Kyowa Kirin Farmacéutica SLU for this study.

Conflicts of interest Antonio Javier Jiménez and Almudena Sanz are employees of Kyowa Kirin Farmacéutica SLU. The authors have no further competing interests.

Open Access This article is distributed under the terms of the Creative Commons Attribution-NonCommercial 4.0 International License (http://creativecommons.org/licenses/by-nc/4.0/), which permits any noncommercial use, distribution, and reproduction in any medium, provided you give appropriate credit to the original author(s) and the source, provide a link to the Creative Commons license, and indicate if changes were made.

\section{References}

1. Mishra S, Bhatnagar S, Chaudhary P, et al. Breakthrough cancer pain: review of prevalence, characteristics and management. Indian J Palliat Care. 2009;15(1):14-8.

2. Portenoy RK, Hagen NA. Breakthrough pain: definition, prevalence and characteristics. Pain. 1990;41(3):273-81.

3. Davies AN, Dickman A, Reid C, et al. The management of cancer-related breakthrough pain: recommendations of a task group of the Science Committee of Association for Palliative Medicine of Great Britain and Ireland. Eur $\mathrm{J}$ Pain. 2009;13(4):331-8. 
4. Caraceni A, Martini C, Zecca E, Working Group of an IASP Task Force on Cancer Pain, et al. Breakthrough pain characteristics and syndromes in patients with cancer pain. An international survey. Palliat Med. 2004;18(3):177-83.

5. Nersesyan H, Slavin KV. Current approach to cancer pain management: availability and implications of different treatment options. Ther Clin Risk Manag. 2007;3(3):381-400.

6. Zeppetella G. Breakthrough pain in cancer patients. Clin Oncol (R Coll Radiol). 2011;23(6):393-8.

7. Pautex S, Vogt-Ferrier N, Zulian GB. Breakthrough pain in elderly patients with cancer: treatment options [review]. Drugs Aging. 2014;31(6):405-11.

8. Lennernäs B, Frank-Lissbrant I, Lennernäs H, et al. Sublingual administration of fentanyl to cancer patients is an effective treatment for breakthrough pain: results from a randomized phase II study. Palliat Med. 2010;24(3):286-93.

9. Rauck RL, Tark M, Reyes E, et al. Efficacy and long-term tolerability of sublingual fentanyl orally disintegrating tablet in the treatment of breakthrough cancer pain. Curr Med Res Opin. 2009;25(12):2877-85

10. Chwieduk CM, McKeage K. Fentanyl sublingual: in breakthrough pain in opioid-tolerant adults with cancer. Drugs. 2010;70(17):2281-8.

11. Nalamachu S, Hassman D, Wallace MS, et al. Long-term effectiveness and tolerability of sublingual fentanyl orally disintegrating tablet for the treatment of breakthrough cancer pain. Curr Med Res Opin. 2011;27(3):519-30.
12. Überall MA, Müller-Schwefe GH. Sublingual fentanyl orally disintegrating tablet in daily practice: efficacy, safety and tolerability in patients with breakthrough cancer pain. Curr Med Res Opin. 2011;27(7):1385-94.

13. Guitart J, Vargas MI, De Sanctis V, et al. Sublingual fentanyl tablets for relief of breakthrough pain in cancer patients and association with quality-of-life outcomes. Clin Drug Investig. 2015;35(12):815-22 (Erratum in: Clin Drug Investig 2016).

14. Ware J Jr, Kosinski M, Keller SD. A 12-Item Short-Form Health Survey: construction of scales and preliminary tests of reliability and validity. Med Care. 1996;34(3):220-33.

15. Schmidt S, Vilagut G, Garin O, et al. Reference guidelines for the 12-Item Short-Form Health Survey version 2 based on the Catalan general population. Med Clin (Barc). 2012;139(14):613-25.

16. Zigmond AS, Snaith RP. The hospital anxiety and depression scale. Acta Psychiatr Scand. 1983;67(6):361-70.

17. Herrero MJ, Blanch J, Peri JM, et al. A validation study of the hospital anxiety and depression scale (HADS) in a Spanish population. Gen Hosp Psychiatry. 2003;25(4):277-83.

18. Jandhyala R, Fullarton JR, Bennett MI. Efficacy of rapid-onset oral fentanyl formulations vs. oral morphine for cancer-related breakthrough pain: a meta-analysis of comparative trials. J Pain Symptom Manage. 2013;46(4):573-580.

19. Brown LF, Kroenke K, Theobald DE, et al. The association of depression and anxiety with health-related quality of life in cancer patients with depression and/or pain. Psychooncology. 2010;19:734-41. 